\title{
27-Hydroxycholesterol, cognition, and brain imaging markers in the FINGER randomized controlled trial
}

\author{
Anna Sandebring-Matton ${ }^{1,2^{*}}$ D, Julen Goikolea ${ }^{1}$, Ingemar Björkhem ${ }^{3}$, Laura Paternain ${ }^{1}$, Nina Kemppainen ${ }^{4,5}$ \\ Tiina Laatikainen 6,7,8, Tiia Ngandu ${ }^{2,8}$, Juha Rinne ${ }^{5}$, Hilkka Soininen ${ }^{9,10}$, Angel Cedazo-Minguez ${ }^{1}$, \\ Alina Solomon $2,9,11+$ and Miia Kivipelto $2,6,11,12+$
}

\begin{abstract}
Background: $27-H y d r o x y c h o l e s t e r o l ~(27-O H)$, the main circulating oxysterol in humans and the potential missing link between peripheral hypercholesterolemia and Alzheimer's disease (AD), has not been investigated previously in relation to cognition and neuroimaging markers in the context of preventive interventions.

Methods: The 2-year Finnish Geriatric Intervention Study to Prevent Cognitive Impairment and Disability (FINGER) included older individuals (60-77 years) at increased risk for dementia but without dementia or substantial cognitive impairment from the general population. Participants were randomized to a multidomain intervention (diet, exercise, cognitive training, and vascular risk management) or control group (general health advice) in a 1:1 ratio. Outcome assessors were masked to group allocation. This FINGER exploratory sub-study included 47 participants with measures of 27-OH, cognition, brain MRI, brain FDG-PET, and PiB-PET. Linear regression models were used to assess the cross-sectional and longitudinal associations between 27-OH, cognition, and neuroimaging markers, considering several potential confounders/intervention effect modifiers.
\end{abstract}

Results: $27-\mathrm{OH}$ reduction during the intervention was associated with improvement in cognition (especially memory). This was not observed in the control group. The intervention reduced $27-\mathrm{OH}$ particularly in individuals with the highest $27-\mathrm{OH}$ levels and younger age. No associations were found between changes in 27-OH levels and neuroimaging markers. However, at baseline, a higher $27-\mathrm{OH}$ was associated with lower total gray matter and hippocampal volume, and lower cognitive scores. These associations were unaffected by total cholesterol levels. While sex seemed to influence associations at baseline, it did not affect longitudinal associations.

Conclusion: 27-OH appears to be a marker not only for dementia/AD risk, but also for monitoring the effects of preventive interventions on cholesterol metabolism.

Trial registration: ClinicalTrials.gov, NCT01041989. Registered on 4 January 2010

Keywords: Cholesterol metabolism, 27-Hydroxycholesterol, Biomarkers, Dementia, Multimodal intervention

\footnotetext{
* Correspondence: anna.matton@ki.se

${ }^{\dagger}$ Alina Solomon and Miia Kivipelto contributed equally to this work.

'Division of Neurogeriatrics, Center for Alzheimer Research, NVS, Karolinska Institutet, Stockholm, Sweden

${ }^{2}$ Division of Clinical Geriatrics, Center for Alzheimer Research, NVS, Karolinska Institutet, Stockholm, Sweden

Full list of author information is available at the end of the article
}

(c) The Author(s). 2021 Open Access This article is licensed under a Creative Commons Attribution 4.0 International License, which permits use, sharing, adaptation, distribution and reproduction in any medium or format, as long as you give appropriate credit to the original author(s) and the source, provide a link to the Creative Commons licence, and indicate if changes were made. The images or other third party material in this article are included in the article's Creative Commons licence, unless indicated otherwise in a credit line to the material. If material is not included in the article's Creative Commons licence and your intended use is not permitted by statutory regulation or exceeds the permitted use, you will need to obtain permission directly from the copyright holder. To view a copy of this licence, visit http://creativecommons.org/licenses/by/4.0/ The Creative Commons Public Domain Dedication waiver (http://creativecommons.org/publicdomain/zero/1.0/) applies to the data made available in this article, unless otherwise stated in a credit line to the data. 


\section{Background}

Alzheimer disease (AD) is the most common cause of dementia and considered a multifactorial disorder with a complex etiology [1, 2]. Lipid metabolism plays an important role in AD [3]. For example, brain cholesterol can affect amyloid precursor protein (APP) processing and amyloid deposition [4], as well as tau phosphorylation [5]. Elevated circulating cholesterol especially in midlife has been linked to increased risk of dementia and $\mathrm{AD}$ in epidemiological studies [6-8], via vascularrelated pathways (e.g., atherosclerosis, stroke, and/or reduced brain blood flow [9]) or potential links to brain amyloid deposition [10, 11]. The association between altered lipid composition and aging, together with the recent progress of lipid research in $\mathrm{AD}$, has led to an increased interest in studying lipid profiles and in particular cholesterol metabolites as fluid biomarkers of AD [12, 13].

The blood-brain barrier separates brain and peripheral cholesterol but allows the flux of its oxidized forms, oxysterols, where 27-hydroxycholesterol $(27-\mathrm{OH})$ is one of the most abundant in the periphery [14]. Oxysterols affect a range of cellular functions and influence multiple physiological processes such as cholesterol metabolism, membrane fluidity, and intracellular signaling pathways. Hence, oxysterols play important roles in pathological conditions such as atherosclerosis, cancer, type 2 diabetes, and neurodegenerative disorders including $\mathrm{AD}$ [15-19]. 27-OH is derived from cholesterol oxidation by CYP27A1 which is expressed by most organs and tissues in humans [20]. High cholesterol in the periphery is associated with high levels of $27-\mathrm{OH}$ in the circulation and thus increased transport of $27-\mathrm{OH}$ into the brain. This transport is also dependent on the high rate of metabolism of $27-\mathrm{OH}$ in the brain as well as by the integrity of the blood-brain barrier [21, 22]. Clinical studies have shown that the levels of $27-\mathrm{OH}$ are increased in both cerebrospinal fluid [22-24] and brain tissue $[25,26]$ of AD patients compared to controls. Further, associations between reduced cognitive performance and high serum levels of $27-\mathrm{OH}$ or ratio of $27-\mathrm{OH}$ to cholesterol have been described in mild cognitive impairment [27] and older ApoE4 carriers [28].

Preclinical studies have pointed out several mechanisms underlying the effects of increased $27-\mathrm{OH}$ on brain function. Notably, CYP27A1-overexpressing mice that produce increased levels of $27-\mathrm{OH}$ develop memory impairment due to, e.g., reduction in neuronal glucose uptake [29], impaired neuronal branching and reduced synaptic density [30], inflammation and impairment of the brain renin-angiotensinogen system [24]. Taken together, the data from preclinical and clinical studies have led to the hypothesis that $27-\mathrm{OH}$ may link $\mathrm{AD}$ and peripheral hypercholesterolemia [16].
While the role of $27-\mathrm{OH}$ in $\mathrm{AD}$ has been studied in animal models and memory clinic patients in relation to cognitive measures and ApoE4 risk allele, no studies have so far investigated $27-\mathrm{OH}$ in the context of preventive interventions. The Finnish Geriatric Intervention Study to Prevent Cognitive Impairment and Disability (FINGER) was a 2-year randomized controlled trial that reported beneficial effects on cognition in an at-risk older general population for a multimodal lifestyle/vascular intervention (combining cardiovascular risk management, diet, exercise, and cognitive training) versus regular health advice control [31]. In the current exploratory study, 27-OH was measured in serum samples from a subset of FINGER participants $(n=47)$, in order to investigate (1) whether the 2-year multidomain lifestyle/vascular intervention had an impact on the $27-\mathrm{OH}$ levels and (2) whether the change in $27-\mathrm{OH}$ during the intervention was related to the change in cognition and dementia-related neuroimaging markers (i.e., gray matter volume, cortical thickness and white matter lesions (WML) on MRI, glucose uptake on FDG-PET, and amyloid load on PiB-PET). In addition, we also investigated the associations of $27-\mathrm{OH}$ with cognition and brain imaging markers at baseline.

\section{Material and methods \\ Study participants}

This exploratory sub-study included 47 FINGER trial participants (21 women and 26 men, mean age $71 \pm 5.1$ years) who underwent both PET and MRI imaging at one of the six trial sites (Turku in southwestern Finland) [32] with serum samples available for 27hydroxycholesterol measurements (Fig. 1). They were selected from the most recently recruited trial participants at the time when MRI/PET resources became available and if there were no contraindications. The demographic, clinical, and cognitive characteristics of these participants have been previously described in detail [32, 33], i.e., they were not different from the rest of the Turku cohort or the rest of the FINGER participants, except for being slightly older (mean $70.8 \pm 5$ years vs $69.3 \pm 4.7$ years for the rest of FINGER participants, due to a later initiation of the recruitment in Turku).

The FINGER population characteristics [34], trial protocol [35], primary findings [31], and neuroimaging sub-study [32, 36] have been previously published. In brief, between September 7, 2009, and November 24, 2011, 2654 individuals were screened and 1260 participants aged 60-77 years from the general population were recruited based on the Cardiovascular Risk Factors, Aging and Dementia (CAIDE) risk score [37] of 6 points or higher, and cognitive performance at the mean level or slightly lower than expected for age according to Finnish population norms for the Consortium to 


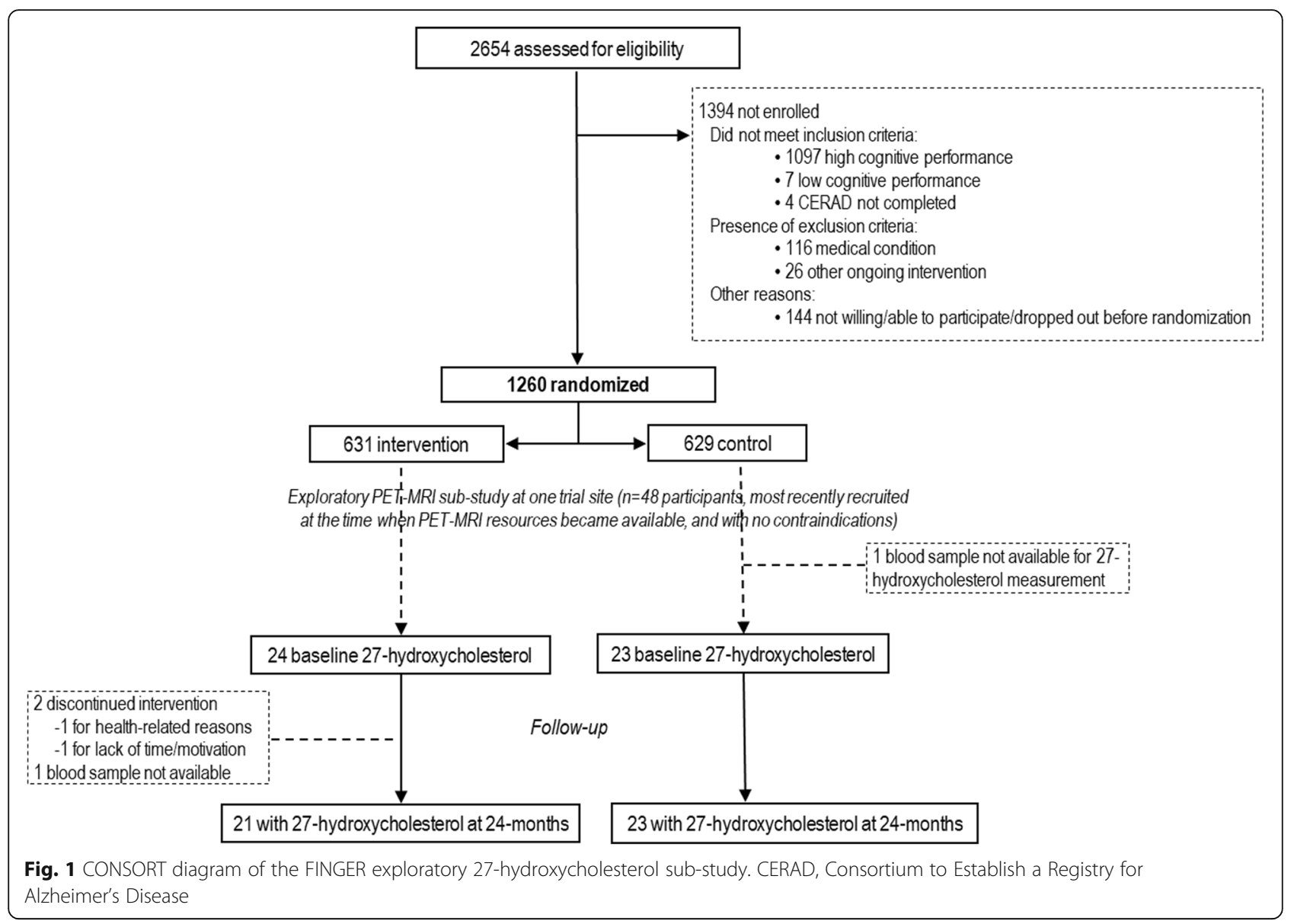

Establish a Registry for Alzheimer's Disease (CERAD) [38]. Individuals were excluded if they had dementia or substantial cognitive impairment, any conditions affecting safe participation/cooperation, or ongoing participation in any other clinical trial.

\section{Intervention}

Participants were randomized 1:1 into the intensive multidomain lifestyle intervention or control group. Outcome assessors were blinded to group allocation, and they were not involved in the intervention activities. The control group received regular health advice. As previously described in detail, the intervention group received nutrition guidance (individual and group sessions with the study nutritionists), physical exercise program at the gym supervised by study physiotherapists, cognitive training (individual computer-based training and group sessions led by study psychologists), and management of metabolic and vascular risk factors [35]. The intervention duration was 2 years.

\section{Cognitive outcomes}

Standard cognitive testing (an extended version of the neuropsychological test battery (NTB) [39]) was conducted for all FINGER participants at the baseline and 12-month and 24-month visits. Pre-specified cognitive outcomes included the NTB total score (composite score based on all 14 tests, calculated as $Z$-scores standardized to the baseline mean and SD), NTB memory, executive functioning, and processing speed domain $Z$ scores calculated as described previously [35]. Higher scores indicated better performance.

\section{MRI and PET imaging}

The subsample of FINGER participants from the Turku site underwent structural 3T MRI (Philips Ingenuity TF PET/MR, Amsterdam, the Netherlands), ${ }^{18}$ F-FDG dynamic PET scan (GE Advance PET scanner in the 3D scanning mode, General Electric Medical Systems, Milwaukee, WI, USA), and ${ }^{11} \mathrm{C}$-Pittsburgh compound $\mathrm{B}$ (PiB)-PET scan (Philips Ingenuity TF PET/MR, Amsterdam, the Netherlands) in connection to both the baseline and 24-month visits. The detailed imaging protocols have been previously published [32, 33].

MRI images were quality checked and read for any abnormalities. Regional brain volumes and cortical thickness were measured using the Freesurfer image analysis suite (version 5.0.3). Brain volumes were normalized to 
the total intracranial volume to adjust for head size. A measure of cortical thickness in $\mathrm{AD}$ signature regions was calculated as the average of cortical thickness in the entorhinal, inferior temporal, middle temporal, and fusiform regions [40]. White matter lesion (WML) volume was measured through the segmentation of white matter hyperintensities on T1 and FLAIR images [41].

For FDG-PET, a dose of ${ }^{18} \mathrm{~F}$-FDG $3.7 \mathrm{MBq} / \mathrm{kg}$ was injected into an antecubital vein as a bolus with a mean dose of $459 \mathrm{MBq}$ (SD $85 \mathrm{MBq}$ ) and flushed with saline. For amyloid PET, on average, $406.3 \mathrm{MBq}$ (SD 107.7 $\mathrm{MBq}$ ) of ${ }^{11} \mathrm{C}$-PiB-PET was injected intravenously. The scans were quantitatively assessed with the automated region of interest analysis. Composite measures were also calculated: for ${ }^{18} \mathrm{~F}$-FDG uptake, the average across the prefrontal, parietal, lateral temporal, precuneus, anterior cingulate, and posterior cingulate cortex; for amyloid deposition, the average across the prefrontal, parietal, lateral temporal, precuneus, anterior cingulate, and posterior cingulate regions of interest. In addition, the amyloid PET scans were visually interpreted by two experienced readers and judged as visually positive or negative after a 2-party consensus agreement.

\section{Blood measurements}

Fasting venous blood samples collected at the baseline and 24-month visits were used in the present study. Serum samples were analyzed for 27-hydroxycholesterol assayed by isotope dilution mass spectrometry using deuteriumlabeled internal standards [42]. For this study, total cholesterol was also assayed by isotope dilution mass spectrometry according to a previously described protocol [43].

\section{Statistical analysis}

For comparisons of the baseline characteristics between the intervention and control groups, $t$ test or $\chi^{2}$ test was used as appropriate. Cross-sectional and longitudinal associations between $27-\mathrm{OH}$ and other variables of interest were investigated using linear regression models, and zero-skewness log transformation was applied to skewed variables.

Change over time in 27-OH, cognitive, MRI, FDGPET, and PIB-PET measures was calculated as the difference between 24-month and baseline values. To study the intervention effect on 27-OH, a linear regression model was conducted with change in $27-\mathrm{OH}$ as an dependent variable and randomization group as an independent variable. To study whether the changes in cognitive, MRI, FDG-PET, and PIB-PET measures (dependent variables) were related to $27-\mathrm{OH}$, the linear regression models included the randomization group, change in $27-\mathrm{OH}$, and their interaction. Potential relevant confounders (age, sex, ApoE4 carrier status, lipidlowering drugs, or total cholesterol change) were also adjusted for additional models.
Analyses of baseline cognitive, MRI, FDG-PET, and PIB-PET measures (as dependent variables) in relation to baseline 27-OH were adjusted for age, self-reported lipid-lowering drugs (and/or diabetes medication for FDG-PET analyses), education (for cognitive measures), and time between blood sample collection and brain scan (for MRI, FDG-PET, and PIB-PET measures) (model 1). Additional adjustments for total cholesterol (model 2) and sex (model 3) were also applied.

The level of significance was set to $p<.05$ in all analyses, and the Stata software, version 14 (StataCorp), was used.

\section{Results}

\section{Population characteristics}

Baseline characteristics of the intervention and control groups are listed in Table 1. Demographic, cognitive, MRI, and PiB-PET measures showed no differences between the groups. The intervention group had significantly lower mean baseline ${ }^{18} \mathrm{~F}$-FDG-PET uptake in the prefrontal and parietal cortex, as well as lower FDG-PET cortical composite measure compared with the control group.

\section{Intervention effects on change in serum $27-\mathrm{OH}$ levels}

Mean $27-\mathrm{OH}$ levels $( \pm \mathrm{SD})$ decreased during 2 years in the intervention group $(-2.8 \pm 55.6 \mathrm{ng} / \mathrm{ml})$ and increased in the control group $(+16.1 \pm 55.3 \mathrm{ng} / \mathrm{ml})$, although the change was not significantly different between groups $(p=0.26)$.

We conducted additional exploratory sub-group analyses of the potential effect of high versus low baseline 27$\mathrm{OH}$ on change over time in 27-OH. The highest (4th) quartile of baseline 27-OH ( $n=11$ participants) was compared with quartiles $1-3$ ( $n=33$ participants) since there is currently no established cutoff for high 27-OH (Fig. 2). In the intervention group, mean $27-\mathrm{OH}$ change was +18 $\mathrm{ng} / \mathrm{ml}$ in quartiles $1-3$, and $-55 \mathrm{ng} / \mathrm{ml}$ in quartile $4(p=$ 0.004 for comparison between quartile 4 and quartiles $1-$ $3)$. In the control group, the mean $27-\mathrm{OH}$ change was + $13 \mathrm{ng} / \mathrm{ml}$ in quartiles $1-3$, and $+26 \mathrm{ng} / \mathrm{ml}$ in quartile 4 . The difference between the change in $27-\mathrm{OH}$ in quartile 4 in the intervention versus the control group was significant $(p=0.03)$, suggesting that the intervention was most efficient in reducing the highest $27-\mathrm{OH}$ levels.

We also investigated if the intervention effect on 27$\mathrm{OH}$ was affected by age at baseline, sex, ApoE4 carrier status, lipid-lowering medication during the intervention, or change in total cholesterol level (Table 2). 27$\mathrm{OH}$ decreased significantly more in the intervention group vs the control group among younger, but not older participants (randomization group $\times$ age interaction, $p=0.01$ ). Sex, ApoE4 carrier status, lipidlowering medication, or total cholesterol change had no significant impact on the intervention effect on $27-\mathrm{OH}$. 
Table 1 Baseline characteristics of the control and intervention groups

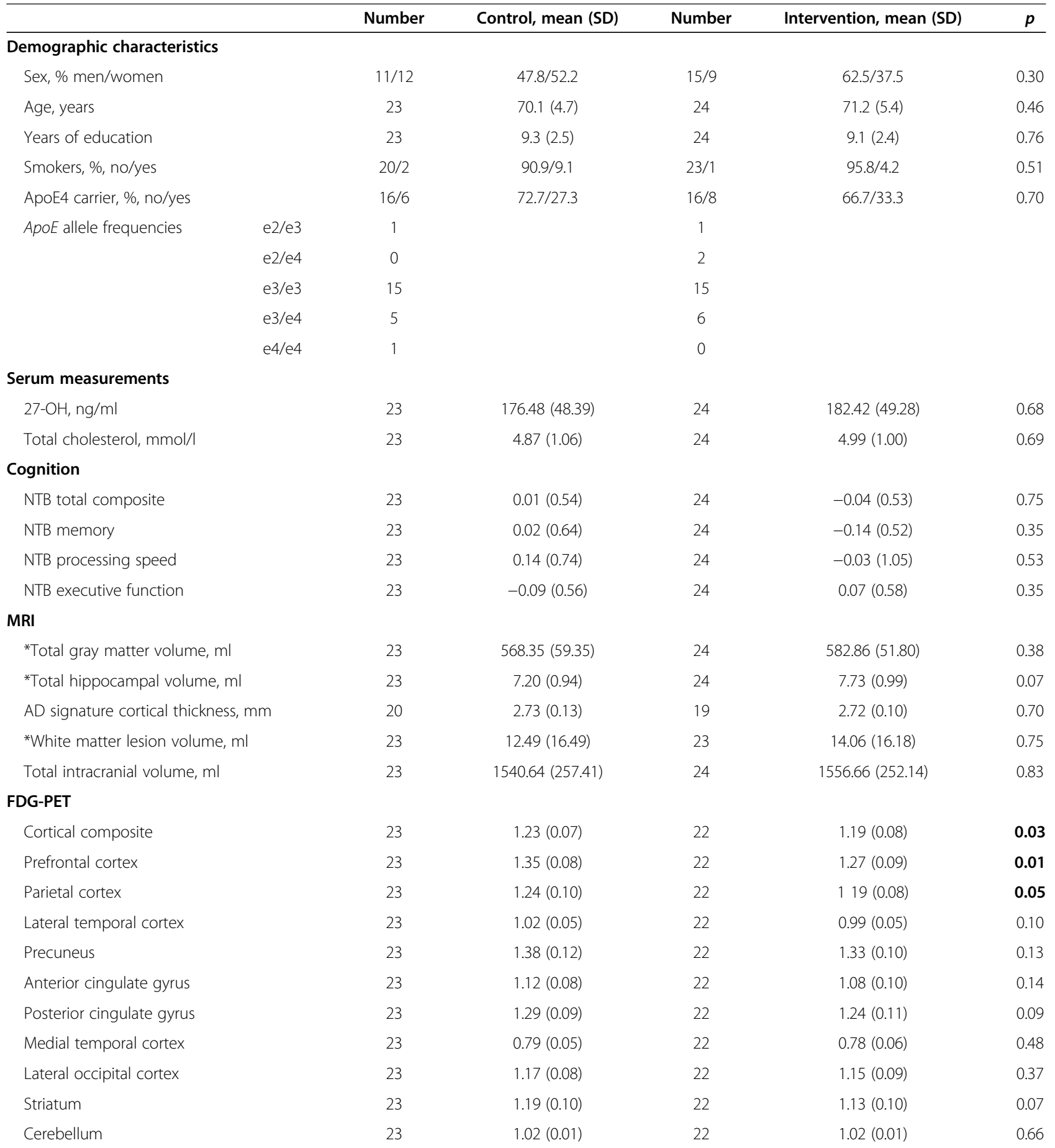

PiB PET

Cortical composite

Values are means \pm SD unless otherwise specified. Between-group differences were analyzed with the chi-square and $t$ tests as appropriate. $p$ value considered significant (marked in bold) if $<0.05$. Cognitive scores are mean values of $Z$-scores of the cognitive tests included in each cognitive outcome, where higher scores indicate better performance. *Brain volumes are shown unadjusted; corresponding $p$ values are shown from analyses with values adjusted for total intracranial volume and zero-skewness log-transformed as appropriate. AD signature cortical thickness was calculated as the average of cortical thickness in the entorhinal, inferior temporal, middle temporal, and fusiform regions. The composite scores for FDG-PET and PiB-PET were determined as the average of the prefrontal, parietal, lateral temporal, anterior cingulate, posterior cingulate, and precuneus ROIs ApoE4, apolipoprotein E4; NTB, neuropsychological test battery; MRI, magnetic resonance imaging; AD, Alzheimer's disease; FDG-PET, 2-deoxy-2fluorine-18fluoro-Dglucose positron emission tomography; PiB, 11 C-Pittsburgh compound B 
\#\#

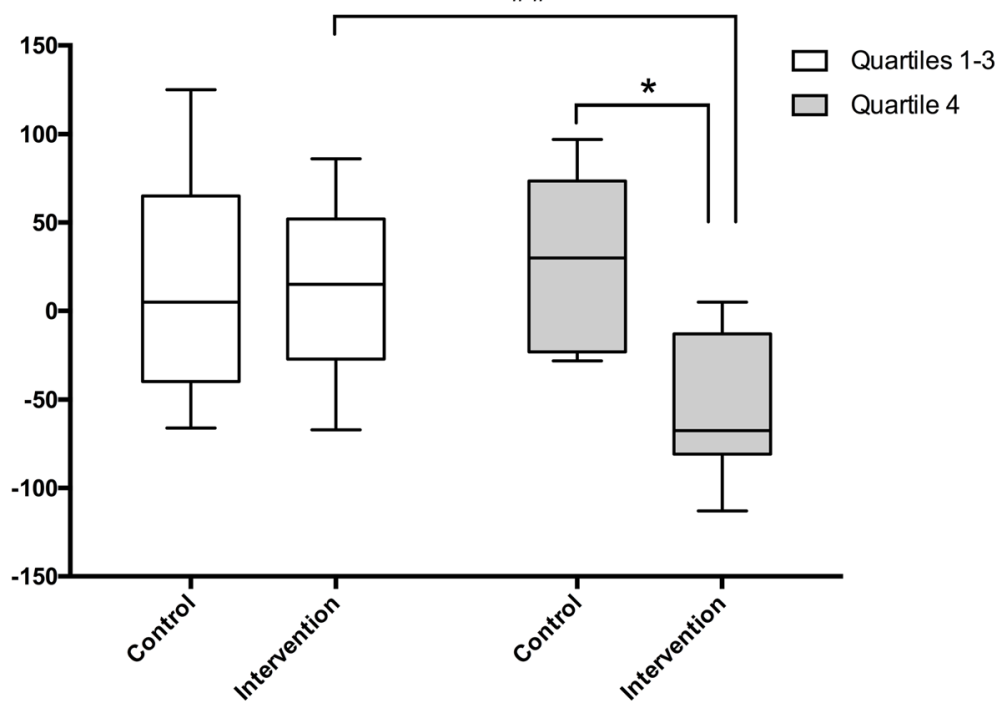

Fig. 2 FINGER intervention effects on the change in 27-OH. Participants were divided into two groups according to their baseline 27-OH levels: the lowest $75 \%$ (quartiles 1-3, white boxplots) and the highest 25\% (quartile 4, gray boxplots). The graph shows the mean change in 27-OH levels $(\mathrm{ng} / \mathrm{ml})$ between the 2-year and baseline measurements. Mann-Whitney non-parametric $U$ test was used to analyze the differences between quartiles $1-3$ and 4 of the intervention group $\left({ }^{\# \#}<0.01\right)$ and between quartile 4 of the control and intervention group $\left({ }^{*} p<0.05\right)$

\section{Longitudinal associations of serum $27-\mathrm{OH}$ levels with cognition and neuroimaging markers}

Table 3 shows associations between the change in $27-\mathrm{OH}$ levels and the change in cognitive scores and brain imaging measures. Overall, change in 27-OH was not associated with the change in cognition or neuroimaging measures. However, there was a significant interaction between the randomization group and change in $27-\mathrm{OH}$ in relation to the change in NTB memory score $(p=0.02)$. A reduction in 27$\mathrm{OH}$ was associated with improvement in NTB memory score in the intervention group $(\beta=-0.54, p=0.01)$, but this association was not found in the control group $(\beta=0.12, p=$ 0.58 ). There was a similar pattern for change in NTB total composite and total gray matter volume, although the randomization group $\times 27-\mathrm{OH}$ interactions showed only non-significant trends ( $p=0.07$ and $p=0.08$, respectively). In stratified analyses, a reduction in $27-\mathrm{OH}$ in the intervention group was significantly related to improvement in NTB total composite score $(\beta=-0.46, p=0.04)$ and a trend for a less pronounced decline in total gray matter volume $(\beta=-0.39$,

Table 2 Intervention effect on 27-OH and impact of age, sex, ApoE4 carrier status, lipid-lowering medication, and change in total cholesterol

\begin{tabular}{|c|c|c|c|}
\hline \multirow[t]{2}{*}{ Factor } & \multicolumn{2}{|c|}{ Difference between intervention and control } & \multirow{2}{*}{$\begin{array}{l}\text { Randomization } \\
\text { group x Factor } \\
\text { interaction, } p\end{array}$} \\
\hline & $N^{*}$ & $\beta(p)$ & \\
\hline \multirow[t]{2}{*}{ Age at baseline } & $<70$ years, $N=22$ & $-0.51(\mathbf{0 . 0 2})$ & 0.01 \\
\hline & $\geq 70$ years, $N=22$ & $0.21(0.34)$ & \\
\hline \multirow[t]{2}{*}{ Sex } & Women, $N=20$ & $-0.06(0.81)$ & 0.52 \\
\hline & Men, $N=24$ & $-0.24(0.26)$ & \\
\hline \multirow[t]{2}{*}{ ApoE4 carrier status } & No, $N=29$ & $-0.13(0.53)$ & 0.96 \\
\hline & Yes, $N=14$ & $-0.13(0.65)$ & \\
\hline \multirow[t]{2}{*}{ Lipid-lowering medication } & No, $N=22$ & $-0.13(0.57)$ & 0.77 \\
\hline & Yes, $N=22$ & $-0.21(0.34)$ & \\
\hline \multirow[t]{2}{*}{ Change in total cholesterol } & Decrease, $N=28$ & $-0.19(0.33)$ & 0.43 \\
\hline & No change/increase, $N=16$ & $-0.18(0.50)$ & \\
\hline
\end{tabular}

Values are standardized $\beta$ coefficients ( $p$ values) from linear regression models with change in 27-OH (difference between 2-year and baseline values) as a dependent variable. Differences between the intervention and control groups are shown from models with randomization group as an independent variable and stratified by each factor (age at baseline, sex, ApoE4 carrier status, lipid-lowering medication, or change in total cholesterol). $p$ value for interaction shown from models including randomization group, factor (as continuous variable for age or total cholesterol change), and the randomization group $x$ factor interaction. $p$ values considered significant (bold) if $<0.05$. ${ }^{*} N$ : participants with available data from both baseline and 2 -year follow-up for each analysis 
Table 3 Longitudinal associations between change in 27-OH and change in cognition and neuroimaging markers

\begin{tabular}{|c|c|c|c|}
\hline & Control, $\beta(p)$ & Intervention, $\beta(p)$ & Interaction, $p$ \\
\hline \multicolumn{4}{|l|}{ Change in cognition } \\
\hline NTB total composite & $0.11(0.62)$ & $-0.46(0.04)$ & 0.07 \\
\hline NTB memory & $0.12(0.58)$ & $-0.54(0.01)$ & 0.02 \\
\hline NTB processing speed & $-0.04(0.85)$ & $0.24(0.31)$ & 0.41 \\
\hline NTB executive function & $0.12(0.59)$ & $-0.31(0.17)$ & 0.18 \\
\hline \multicolumn{4}{|l|}{ Change in MRI } \\
\hline Total gray matter volume & $0.18(0.45)$ & $-0.39(0.10)$ & 0.08 \\
\hline Hippocampal volume & $0.06(0.79)$ & $-0.28(0.25)$ & 0.30 \\
\hline AD signature cortical thickness & $0.05(0.84)$ & $-0.20(0.42)$ & 0.50 \\
\hline White matter lesion volume & $-0.05(0.82)$ & $-0.05(0.85)$ & 0.97 \\
\hline \multicolumn{4}{|l|}{ Change in FDG-PET } \\
\hline Cortical composite & $0.24(0.32)$ & $0.01(0.97)$ & 0.42 \\
\hline Prefrontal cortex & $0.08(0.74)$ & $0.04(0.89)$ & 0.85 \\
\hline Parietal cortex & $0.21(0.36)$ & $0.11(0.70)$ & 0.68 \\
\hline Lateral temporal cortex & $0.20(0.40)$ & $-0.03(0.91)$ & 0.46 \\
\hline Precuneus & $0.23(0.33)$ & $-0.04(0.89)$ & 0.41 \\
\hline Anterior cingulate gyrus & $0.25(0.29)$ & $-0.05(0.85)$ & 0.34 \\
\hline Posterior cingulate gyrus & $0.26(0.27)$ & $0.00(0.99)$ & 0.34 \\
\hline Medial temporal cortex & $0.25(0.30)$ & $0.13(0.64)$ & 0.59 \\
\hline Lateral occipital cortex & $0.23(0.32)$ & $-0.28(0.29)$ & 0.18 \\
\hline Striatum & $0.38(0.10)$ & $-0.08(0.77)$ & 0.15 \\
\hline Cerebellum & $-0.01(0.97)$ & $0.22(0.41)$ & 0.50 \\
\hline \multicolumn{4}{|l|}{ Change in PIB-PET } \\
\hline Cortical composite & $-0.04(0.87)$ & $-0.10(0.69)$ & 0.81 \\
\hline
\end{tabular}

Values are standardized $\beta$ coefficients ( $p$ values) from linear regression models with change in cognitive and neuroimaging measures (left column) as dependent variables and change in $27-\mathrm{OH}$ as an independent variable. Change in all measures was calculated as the difference between 2-year and baseline values. Values for the control and intervention groups are shown from analyses stratified by the randomization group. $p$ value for interaction is shown from models including change in $27-\mathrm{OH}$, randomization group, and their interaction. $p$ value considered significant (bold) if $<0.05$. AD signature cortical thickness was calculated as the average of cortical thickness in the entorhinal, inferior temporal, middle temporal, and fusiform regions. The changes in composite scores for FDG-PET and PiB-PET were determined as the average change of the prefrontal, parietal, lateral temporal, anterior cingulate, posterior cingulate, and precuneus ROIs

NTB, neuropsychological test battery; MRI, magnetic resonance imaging; AD, Alzheimer's disease; FDG-PET, 2-deoxy-2-fluorine-18fluoro-D-glucose positron emission tomography; PiB, 11 C-Pittsburgh compound B

$p=0.10)$. These associations were not found in the control group.

Additional adjustment for age at baseline, sex, lipidlowering drugs, or change in total cholesterol did not change the observed associations (results not shown), except for the change in NTB memory score where the randomization group $\times 27-\mathrm{OH}$ change interaction became a non-significant trend $(p=0.09)$ after adjusting for age.

\section{Baseline associations of serum 27-OH levels with cognition and neuroimaging markers}

Association between 27-OH, cognition, and neuroimaging markers at baseline are shown in Table 4. Higher 27-OH was associated to lower NTB total composite $(\beta=-0.28, p=0.04)$ and NTB memory scores $(\beta=-$ $0.36, p=0.01)$ in model 1 . These associations remained significant after adjusting for total cholesterol levels $(\beta=$
$-0.42, p=0.01$ for NTB total composite and $\beta=-0.59$, $p=0.00$ for NTB memory) (model 2). After further adjusting for sex (model 3), there was only a nonsignificant trend for association with NTB memory $(\beta=$ $-0.27, p=0.08)$, and the association with NTB total composite was no longer statistically significant $(\beta=-$ $0.22, p=0.12$ ). No significant associations were found with processing speed or executive functioning scores in any of the models.

Higher 27-OH was related to lower hippocampal volume $(\beta=-0.31, p=0.04)$, with a non-significant trend for lower total gray matter volume $(\beta=-0.27, p=0.09)$ (model 1). These associations were significant after adjusting for total cholesterol (model 2) $(\beta=-0.48, p=$ 0.00 and $\beta=-0.45, p=0.01$, respectively), but not after additional adjustment for sex (model 3$)(\beta=-0.14, p=$ 0.31 and $\beta=-0.08, p=0.61$ respectively). No significant 
Table 4 Cross-sectional associations between 27-OH, cognition, and neuroimaging markers at baseline

\begin{tabular}{|c|c|c|c|}
\hline & Model $1, \beta(p)$ & Model $2, \beta(p)$ & Model $3, \beta(p)$ \\
\hline \multicolumn{4}{|l|}{ Cognition } \\
\hline NTB total composite & $-0.28(0.04)$ & $-0.42(0.01)$ & $-0.22(0.12)$ \\
\hline NTB memory & $-0.36(0.01)$ & $-0.59(0.00)$ & $-0.27(0.08)$ \\
\hline NTB processing speed & $-0.20(0.20)$ & $-0.27(0.14)$ & $-0.16(0.34)$ \\
\hline NTB executive function & $-0.09(0.53)$ & $-0.08(0.62)$ & $-0.08(0.57)$ \\
\hline \multicolumn{4}{|l|}{ MRI } \\
\hline Total gray matter volume & $-0.27(0.09)$ & $-0.45(0.01)$ & $-0.08(0.61)$ \\
\hline Hippocampal volume & $-0.31(0.04)$ & $-0.48(0.00)$ & $-0.14(0.31)$ \\
\hline AD signature cortical thickness & $0.01(0.98)$ & $0.01(0.96)$ & $-0.15(0.42)$ \\
\hline White matter lesion volume & $0.24(0.13)$ & $0.31(0.10)$ & $0.21(0.22)$ \\
\hline \multicolumn{4}{|l|}{ FDG-PET } \\
\hline Cortical composite & $-0.22(0.16)$ & $-0.27(0.11)$ & $-0.08(0.66)$ \\
\hline Prefrontal cortex & $-0.03(0.86)$ & $-0.04(0.82)$ & $-0.08(0.66)$ \\
\hline Parietal cortex & $-0.27(0.09)$ & $-0.30(0.09)$ & $-0.16(0.38)$ \\
\hline Lateral temporal cortex & $-0.21(0.21)$ & $-0.27(0.14)$ & $-0.13(0.48)$ \\
\hline Precuneus & $-0.28(0.10)$ & $-0.32(0.09)$ & $-0.11(0.55)$ \\
\hline Anterior cingulate gyrus & $-0.17(0.27)$ & $-0.20(0.24)$ & $-0.09(0.62)$ \\
\hline Posterior cingulate gyrus & $-0.20(0.23)$ & $-0.30(0.08)$ & $-0.02(0.91)$ \\
\hline Medial temporal cortex & $-0.02(0.90)$ & $-0.14(0.44)$ & $-0.05(0.80)$ \\
\hline Lateral occipital cortex & $-0.31(0.07)$ & $-0.29(0.11)$ & $-0.40(0.04)$ \\
\hline Striatum & $-0.28(0.10)$ & $-0.26(0.15)$ & $-0.27(0.16)$ \\
\hline Cerebellum & $0.00(0.98)$ & $-0.05(0.81)$ & $0.21(0.25)$ \\
\hline \multicolumn{4}{|l|}{ PIB-PET } \\
\hline Cortical composite & $-0.06(0.73)$ & $-0.08(0.67)$ & $-0.02(0.91)$ \\
\hline
\end{tabular}

Values are standardized $\beta$ coefficients ( $p$ values) from linear regression models with cognitive and neuroimaging measures (left column) as dependent variables and 27- $\mathrm{OH}$ as an independent variable. Model 1 was adjusted for age, self-reported lipid-lowering medication (and/or diabetes medication for FDG-PET measures), education (for cognitive measures), and time between blood sample collection and brain scan (for MRI, FDG- and PIB-PET measures). Model 2 was additionally adjusted for serum total cholesterol. Model 3 was additionally adjusted for sex. $p$ values considered significant (bold) if $<0.05$. AD signature cortical thickness was calculated as the average of cortical thickness in the entorhinal, inferior temporal, middle temporal, and fusiform regions. FDG-PET and PiB-PET cortical composite scores were calculated as the average across prefrontal, parietal, lateral temporal, precuneus, anterior cingulate, and posterior cingulate cortex

NTB, neuropsychological test battery; MRI, Magnetic Resonance Imaging; AD, Alzheimer's disease; FDG-PET, 2-deoxy-2-fluorine-18fluoro-D-glucose Positron emission tomography; PiB, 11 C-Pittsburgh compound B

associations were found with WML volume or cortical thickness in AD signature areas.

There was a consistent pattern of association between higher 27-OH and lower ${ }^{18}$ F-FDG uptake on PET scans across several brain regions, although the associations did not reach statistical significance. Adjustment for ApoE4 carrier status did not influence the associations seen (data not shown).

Given the impact of sex on the regression models, and known sex differences in peripheral 27-OH levels, we also compared baseline characteristics between men and women. As expected, $27-\mathrm{OH}$ was higher for men (194 \pm $52 \mathrm{ng} / \mathrm{ml})$ vs women $(162 \pm 38 \mathrm{ng} / \mathrm{ml}), p=0.02$. Total gray matter volume and hippocampal volume (adjusted for head size) were significantly larger in women compared to men ( 0.40 vs $0.36, p=0.00$ and 0.0054 vs $0.0046, p=0.01)$. No differences were found for total cholesterol levels, cognitive, demographic, or other neuroimaging measures (results not shown).

\section{Discussion}

Peripheral hypercholesterolemia and brain cholesterol metabolism are features known to influence both the risk and the progression of the disease in $\mathrm{AD}$ and other dementia-related disorders [16, 44]. 27-OH is a key link between the peripheral and brain cholesterol pools and associations between 27-OH, cognition and related biomarkers have been previously analyzed in observational studies [22, 24]. In this exploratory FINGER sub-study, we investigated for the first time the effect of a dementia preventive intervention on $27-\mathrm{OH}$, and its relation to the change in cognition and neuroimaging markers. A reduction in 27-OH during the multidomain lifestyle/ vascular intervention was associated with improvement 
in cognition (especially memory, with a trend for overall cognitive performance). $27-\mathrm{OH}$ reduction during the intervention also tended to be related to less decline in total gray matter volume. These associations were not observed in the control group, where 27-OH levels tended to increase over time. The multidomain lifestyle/ vascular intervention seemed to reduce $27-\mathrm{OH}$ particularly in individuals with the highest $27-\mathrm{OH}$ levels at baseline. Thus, our findings suggest that benefits on cognition and structural brain changes may require more intensive modifications of an individual's cholesterol metabolites. These findings are especially important given that the main FINGER trial reported no significant intervention benefits on total cholesterol [31]. 27-OH may be a more sensitive marker for monitoring the benefits of lifestyle/vascular interventions on cholesterol metabolism. Reduction in 27-OH may also represent one possible mechanism behind the previously reported intervention benefits on cognition [31].

Interestingly, age (but not sex, lipid-lowering medication, or change in total cholesterol) had a significant impact on the FINGER intervention effect on 27-OH. It is not fully clear why younger individuals had more intervention benefits on $27-\mathrm{OH}$ reduction compared with older individuals. Participants in the highest 27-OH quartile at baseline tended to be younger (mean 68.4 vs 71.4 years for the lower $27-\mathrm{OH}$ quartiles), which may at least partly explain this finding. Future studies on a larger sample are needed to understand more about this association.

We found no statistically significant longitudinal associations between change in $27-\mathrm{OH}$ and change in other MRI, FDG-PET, or PiB-PET measures. FINGER participants were older individuals with vascular/lifestyle risk factors, but without substantial cognitive impairment or dementia, i.e., the preventive intervention was started early, before the occurrence of extensive brain pathology. Overall, changes in neuroimaging parameters in this population were relatively small during 2 years [36]. In addition, the multidomain lifestyle/ vascular intervention was designed to target multiple risk factors and disease-related mechanisms simultaneously, i.e., it did not specifically target amyloid pathology. If $27-\mathrm{OH}$ reduction is a possible mechanism for intervention benefits on cognition, this mechanism may not necessarily be related to amyloid accumulation. Ongoing extended FINGER follow-ups will provide further data on longer-term changes in neuroimaging markers and how they relate to changes in cholesterol metabolism.

Previous observational studies in patients with mild cognitive impairment and elderly ApoE4 carriers have reported associations between elevated $27-\mathrm{OH}$ and poorer cognitive performance $[27,28]$. The present study investigated for the first time serum 27-OH, cognition, and related neuroimaging markers in at-risk older individuals without dementia or substantial cognitive impairment. In cross-sectional analyses at baseline, higher levels of 27-OH were associated with lower scores on global cognition and memory, and lower total gray matter and hippocampal volumes. These associations were influenced by sex, possibly due to men having higher 27-OH compared with women [45], e.g., because of generally higher cholesterol levels and/or reduced rate of metabolism. Other possible explanations may be sex differences in BBB permeability [46] allowing a higher proportion of $27-\mathrm{OH}$ to enter the brain in men [47] or other sex-related factors not accounted for in this study.

However, the associations between $27-\mathrm{OH}$, cognition, and MRI measures were not substantially affected by serum total cholesterol levels. Findings from the present study thus support the notion that 27-OH acts independently of cholesterol on brain functioning. Potential mechanisms have been described in in vitro and in vivo experiments in models with elevated 27-OH. Along with memory impairment, CYP27A1 tg mice have an upregulated brain renin-angiotensin system [24] and reduction in dendritic arborization, spine density of the hippocampus [30], and levels of long-term memory-related protein Arc (activity-regulated cytoskeleton-associated protein). Importantly, CYP27A1 knock-out mice show less memory impairment upon high-fat diet and restoration of cholesterol-decreased levels of Arc in comparison with wild-type controls [48], a finding suggesting that $27-\mathrm{OH}$ is an important factor mediating the risk of cognitive impairment caused by dietary cholesterol. A previous study on cognitively impaired patients showed that high 27$\mathrm{OH}$ levels in CSF were associated with reduced brain glucose uptake [29]. These patients did not have high cholesterol levels nor lipid-lowering medications, suggesting that the associations were independent of total cholesterol levels but rather related to a high cholesterol metabolic rate.

CYP27A1, the enzyme responsible for converting cholesterol to $27-\mathrm{OH}$, was recently explored as a new target for breast cancer adjuvant therapy. The approved drug anastrazole was shown to successfully inhibit CYP27A1 and thereby lower 27-OH levels without altering cholesterol levels in mice [49]. Pharmaceutical adjustment of oxysterols, and in particular 27-OH in combination with a lifestyle intervention, may be an attractive new avenue for dementia prevention. Given the important role of CYP27A1 for the formation of bile acids, altering the levels of 27-OH must however be monitored carefully.

In this exploratory FINGER sub-study, although there seemed to be a consistent pattern of association between higher serum 27-OH and lower brain glucose uptake, the associations did not reach statistical significance. 
This could be due to two main reasons: (i) the small sample size and thus limited statistical power of this study and (ii) FINGER included at-risk participants without dementia or substantial impairment, while a more substantial and widespread impact of $27-\mathrm{OH}$ on glucose uptake in the brain may become more evident in later stages of disease progression. In the future, follow-up studies on larger cohorts including the prodromal AD stage would be helpful to further investigate the role of $27-\mathrm{OH}$ on glucose brain uptake in disease progression.

27-OH levels were not significantly related to amyloid accumulation on PiB-PET scans at baseline in the present study. Previous experimental studies have provided conflicting data on amyloid and $27-\mathrm{OH}$, with $27-$ $\mathrm{OH}$ reported to have a slight inhibitory effect on APP secretion and amyloid production [50], but also to increase BACE1, APP, and A $\beta$ levels [51]. No correlation was found between CSF levels of $27-\mathrm{OH}$ and $\mathrm{A} \beta 42$, but with plasma 27-OH and CSF soluble APP [52]. The relationship between amyloid and $27-\mathrm{OH}$ in at-risk states and early disease stages should be further investigated in larger cohorts.

The main strengths of the present study are the randomized controlled design, multidomain lifestyle/vascular intervention with high relevance for peripheral lipid metabolism, and comprehensive repeated assessments of $27-\mathrm{OH}$, cognition, and related neuroimaging markers during 2 years.

\section{Limitations}

The main limitation is the small sample size affecting statistical power. Because all analyses are post hoc and no correction for multiple testing has been applied, findings should be considered exploratory and will need to be verified in further studies with larger sample sizes.

\section{Conclusions}

In conclusion, a reduction in $27-\mathrm{OH}$ during the multidomain lifestyle/vascular intervention was associated with improvement in cognition (especially memory). This was not observed in the control group. The intervention seemed to reduce 27-OH particularly in individuals with the highest 27-OH levels and younger age. Change in 27$\mathrm{OH}$ was not significantly associated with the change in neuroimaging markers during 2 years. However, higher $27-\mathrm{OH}$ at baseline was associated with lower total gray matter and hippocampal volume and lower cognitive test scores. These associations were mostly unaffected by total cholesterol levels. While sex seemed to impact most associations at baseline, it did not affect longitudinal associations. 27-OH should be further investigated not only as a potential biomarker for dementia/AD risk, but also as a potential marker for monitoring the effect of dementia preventive interventions.

\begin{abstract}
Abbreviations
27-OH: 27-Hydroxycholesterol; FINGER: Finnish Geriatric Intervention Study to Prevent Cognitive Impairment and Disability; AD: Alzheimer's disease; WML: White matter lesions; NTB: Neuropsychological test battery; ApoE: Apolipoprotein E; MRI: Magnetic resonance imaging; PET: Positron emission tomography; PiB: C-Pittsburgh compound B
\end{abstract}

\begin{abstract}
Acknowledgements
The authors would like to thank the entire FINGER study group for all their valuable contributions to the design and implementation of the FINGER trial. The authors would also like to thank the FINGER participants for their time and efforts.
\end{abstract}

\section{Authors' contributions}

ASM, IB, TL, TN, JR, HS, ACM, AS, and MK designed the research. ASM, JG, IB, $L P, N K, J R, H S$, and TN acquired the data. ASM, JG, TN, NK, AS, and MK collected the data. ASM, TN, JG, AS, and MK drafted the manuscript. The authors read and approved the final manuscript.

\section{Funding}

This research was supported by the Swedish Research Council, Center for Innovative Medicine (CIMED) at Karolinska Institutet, Margareta af Ugglas foundation, Alzheimerfonden Sweden, Region Stockholm (ALF, NSV), Knut and Alice Wallenberg Foundation, Stiftelsen Stockholms sjukhem, Konung Gustaf V:s och Drottning Victorias Frimurarstiftelse, Gun och Bertil Stohnes Stiftelse, the Karolinska Institutet fund for geriatric research, Stiftelsen Gamla Tjänarinnor, Alzheimer's Research \& Prevention Foundation, Academy of Finland, Finnish Social Insurance Institution, Finnish Ministry of Education and Culture, Juho Vainio Foundation (Finland), Joint Program of

Neurodegenerative Disorders - prevention (EURO-FINGERS), European Research Council grant 804371, Yrjö Jahnsson Foundation (Finland), and Finnish Cultural Foundation. Open Access funding provided by Karolinska Institute.

\section{Availability of data and materials}

Data are available upon request. Public deposition of the de-identified data set is not possible due to legal and ethical reasons, and complete deidentification is not possible as this investigation is part of an ongoing study. The study participants gave informed consent which includes data use only under a confidentiality agreement. Further, the data contains a large amount of sensitive information, and public data deposition may pose privacy concerns. Those fulfilling the requirements for viewing confidential data as required by the Finnish law and the Finnish National Institute for Health and Welfare are able to access the data after completion of the material transfer agreement. Requests may be addressed to kirjaamo@thl.fi.

Shared data relevant to the present study will encompass the data dictionary and pseudonymized participant data only. Study protocol including statistical analysis plan for the FINGER trial has been previously published [31, 35].

\section{Ethics approval and consent to participate}

The FINGER study adhered to the Declaration of Helsinki and was approved by the Coordinating Ethics Committee of the Hospital District of Helsinki and Uusimaa. Participants gave written informed consent at the screening and baseline visits. Participants in the FINGER neuroimaging sub-studies also gave consent for the brain scans.

\section{Consent for publication \\ Not applicable}

\section{Competing interests}

The authors declare that they have no competing interests.

\section{Author details}

${ }^{1}$ Division of Neurogeriatrics, Center for Alzheimer Research, NVS, Karolinska Institutet, Stockholm, Sweden. ${ }^{2}$ Division of Clinical Geriatrics, Center for Alzheimer Research, NVS, Karolinska Institutet, Stockholm, Sweden. ${ }^{3}$ Division of Clinical Chemistry, Department of Laboratory Medicine, Karolinska University Hospital, Stockholm, Sweden. ${ }^{4}$ Division of Clinical Neurosciences, Turku University Hospital, Turku, Finland. ${ }^{5}$ Turku PET Centre, University of Turku and Turku University Hospital, Turku, Finland. ${ }^{6}$ Institute of Public Health and Clinical Nutrition, University of Eastern Finland, Kuopio, Finland. ${ }^{7}$ Joint 
Municipal Authority for North Karelia Social and Health Services, Joensuu, Finland. ${ }^{8}$ Public Health Promotion Unit, Department of Public Health Solutions, Finnish Institute for Health and Welfare, Helsinki, Finland. ${ }^{9}$ Institute of Clinical Medicine/Neurology, University of Eastern Finland, Kuopio, Finland.

${ }^{10}$ Neurocenter, Neurology Kuopio University Hospital, Kuopio, Finland. ${ }^{11}$ Ageing Epidemiology (AGE) Research Unit, School of Public Health, Imperial College London, London, UK. ${ }^{12}$ Theme Aging, Karolinska University Hospital, Stockholm, Sweden.

\section{Received: 28 September 2020 Accepted: 15 February 2021} Published online: 06 March 2021

\section{References}

1. lqbal K, Grundke-lqbal I. Alzheimer's disease, a multifactorial disorder seeking multitherapies. Alzheimers Dement. 2010;6(5):420-4.

2. Jack CRJ, Bennett DA, Blennow K, Carrillo MC, Dunn B, Haeberlein SB, et al. NIA-AA Research Framework: toward a biological definition of Alzheimer's disease. Alzheimers Dement. 2018;14(4):535-62.

3. Shinohara M, Sato N. The roles of apolipoprotein E, lipids, and glucose in the pathogenesis of Alzheimer's disease. Adv Exp Med Biol. 2019;1128:85101.

4. Sun JH, Yu JT, Tan L. The role of cholesterol metabolism in Alzheimer's disease. Mol Neurobiol. 2015;51(3):947-65.

5. Fan QW, Yu W, Senda T, Yanagisawa K, Michikawa M. Cholesteroldependent modulation of tau phosphorylation in cultured neurons. J Neurochem. 2001;76(2):391-400 Available from: http://www.ncbi.nlm.nih. gov/pubmed/11208902.

6. Hooshmand B, Polvikoski T, Kivipelto M, Tanskanen M, Myllykangas L, Makela M, et al. CAIDE Dementia Risk Score, Alzheimer and cerebrovascular pathology: a population-based autopsy study. J Intern Med. 2018;283(6): 597-603.

7. Solomon A, Kivipelto M, Wolozin B, Zhou J, Whitmer RA. Midlife serum cholesterol and increased risk of Alzheimer's and vascular dementia three decades later. Dement Geriatr Cogn Disord. 2009;28(1):75-80 Available from: http://www.ncbi.nlm.nih.gov/pubmed/19648749.

8. Anstey KJ, Ashby-Mitchell K, Peters R. Updating the evidence on the association between serum cholesterol and risk of late-life dementia: review and meta-analysis. J Alzheimers Dis. 2017;56(1):215-28.

9. Hamel E, Royea J, Ongali B, Tong XK. Neurovascular and cognitive failure in Alzheimer's disease: benefits of cardiovascular therapy. Cell Mol Neurobiol. 2016;36(2):219-32.

10. Pappolla MA, Bryant-Thomas TK, Herbert D, Pacheco J, Fabra Garcia M, Manjon $\mathrm{M}$, et al. Mild hypercholesterolemia is an early risk factor for the development of Alzheimer amyloid pathology. Neurology. 2003;61(2):199205.

11. Reed B, Villeneuve S, Mack W, DeCarli C, Chui HC, Jagust W. Associations between serum cholesterol levels and cerebral amyloidosis. JAMA Neurol. 2014;71(2):195-200.

12. Zarrouk A, Vejux A, Mackrill J, O'Callaghan Y, Hammami M, O'Brien N, et al. Involvement of oxysterols in age-related diseases and ageing processes. Ageing Res Rev. 2014;18:148-62.

13. Zarrouk A, Debbabi M, Bezine M, Karym EM, Badreddine A, Rouaud O, et al. Lipid biomarkers in Alzheimer's disease. Curr Alzheimer Res. 2018;15(4):30312.

14. Loera-Valencia R, Goikolea J, Parrado-Fernandez C, Merino-Serrais P, Maioli S. Alterations in cholesterol metabolism as a risk factor for developing Alzheimer's disease: potential novel targets for treatment. J Steroid Biochem Mol Biol. 2019;190:104-14.

15. Duc D, Vigne S, Pot C. Oxysterols in Autoimmunity. Int J Mol Sci. 2019; 20(18):4522.

16. Björkhem I, Cedazo-minguez A, Leoni V, Meaney S. Oxysterols and neurodegenerative diseases. Mol Aspects Med. 2009;30(3):171-9 Available from: https://doi.org/10.1016/j.mam.2009.02.001.

17. Leoni V. Oxysterols as markers of neurological disease--a review. Scand J Clin Lab Invest. 2009;69(1):22-5 Available from: http://www.ncbi.nlm.nih. gov/pubmed/19199127.

18. Griffiths WJ, Wang Y. Oxysterol research: a brief review. Biochem Soc Trans. 2019;47(2):517-26.

19. Brown RB. Phospholipid packing defects and oxysterols in atherosclerosis: dietary prevention and the French paradox. Biochimie. 2019;167:145-51.
20. Marwarha G, Ghribi O. Does the oxysterol 27-hydroxycholesterol underlie Alzheimer's disease-Parkinson's disease overlap? Exp Gerontol. 2015;68:13-8.

21. Ali Z, Heverin M, Olin M, Acimovic J, Lövgren-Sandblom A, Shafaati M, et al. On the regulatory role of side-chain hydroxylated oxysterols in the brain. Lessons from CYP27A1 transgenic and Cyp27a1 -/- mice 1. J Lipid Res. 2013;54(4):1033-43.

22. Leoni V, Masterman T, Mousavi FS, Wretlind B, Wahlund LO, Diczfalusy U, et al. Diagnostic use of cerebral and extracerebral oxysterols. Clin Chem Lab Med. 2004;42(2):186-91.

23. Wang HL, Wang YY, Liu XG, Kuo SH, Liu N, Song QY, et al. Cholesterol, 24hydroxycholesterol, and 27-hydroxycholesterol as surrogate biomarkers in cerebrospinal fluid in mild cognitive impairment and Alzheimer's disease: a meta-analysis. J Alzheimers Dis. 2016;51(1):45-55.

24. Mateos L, Ismail M-A-M, Gil-Bea F-J, Leoni V, Winblad B, Björkhem I, et al. Upregulation of brain renin angiotensin system by 27-hydroxycholesterol in Alzheimer's disease. J Alzheimers Dis. 2011;24(4):669-79 Available from: http://www.ncbi.nlm.nih.gov/pubmed/21297254

25. Heverin M, Bogdanovic N, Lutjohann D, Bayer T, Pikuleva I, Bretillon L, et al. Changes in the levels of cerebral and extracerebral sterols in the brain of patients with Alzheimer's disease. J Lipid Res. 2004;45(1):186-93.

26. Testa G, Staurenghi E, Zerbinati C, Gargiulo S, Iuliano L, Giaccone G, et al. Changes in brain oxysterols at different stages of Alzheimer's disease: their involvement in neuroinflammation. Redox Biol. 2016;10:24-33.

27. Liu Q, An Y, Yu H, Lu Y, Feng L, Wang C, et al. Relationship between oxysterols and mild cognitive impairment in the elderly: a case - control study; 2016. p. 1-6.

28. van den Kommer TN, Dik MG, Comijs HC, Fassbender K, Lutjohann D, Jonker C. Total cholesterol and oxysterols: early markers for cognitive decline in elderly? Neurobiol Aging. 2009;30(4):534-45

29. Ismail M-A-M, Mateos L, Maioli S, Merino-Serrais P, Ali Z, Lodeiro M, et al. 27Hydroxycholesterol impairs neuronal glucose uptake through an IRAP/ GLUT4 system dysregulation. J Exp Med. 2017:jem.20160534 Available from: http://www.jem.org/lookup/doi/10.1084/jem.20160534.

30. Merino-Serrais $P$, Loera-Valencia R, Rodriguez-Rodriguez P, ParradoFernandez C, Ismail MA, Maioli S, et al. 27-Hydroxycholesterol induces aberrant morphology and synaptic dysfunction in hippocampal neurons. Cereb Cortex. 2018;(November 2018):429-46 Available from: https://aca demic.oup.com/cercor/advance-article/doi/10.1093/cercor/bhy274/5158244.

31. Ngandu T, Lehtisalo J, Solomon A, Levälahti E, Ahtiluoto S, Antikainen R, et al. A 2 year multidomain intervention of diet, exercise, cognitive training, and vascular risk monitoring versus control to prevent cognitive decline in at-risk elderly people (FINGER): a randomised controlled trial. Lancet. 2015; 385(9984):2255-63.

32. Kemppainen N, Johansson J, Teuho J, Parkkola R, Joutsa J, Ngandu T, et al. Brain amyloid load and its associations with cognition and vascular risk factors in FINGER study. Neurology. 2017. https://doi.org/10.1212/WNL. 0000000000004827 Available from: http://www.neurology.org/lookup/doi/1 0.1212 WNL.0000000000004827.

33. Stephen R, Liu Y, Ngandu T, Rinne JO, Kemppainen N, Parkkola R, et al. Associations of CAIDE Dementia Risk Score with MRI, PIB-PET measures, and cognition. J Alzheimers Dis. 2017;59(2):695-705.

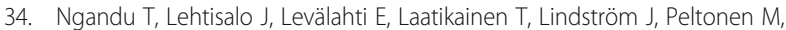
et al. Recruitment and baseline characteristics of participants in the Finnish Geriatric Intervention Study to Prevent Cognitive Impairment and Disability (FINGER)-a randomized controlled lifestyle trial. Int J Environ Res Public Health. 2014;11(9):9345-60 Available from: http://www.ncbi.nlm.nih.gov/ pubmed/25211775

35. Kivipelto M, Solomon A, Ahtiluoto S, Ngandu T, Lehtisalo J, Antikainen R, et al. The Finnish Geriatric Intervention Study to Prevent Cognitive Impairment and Disability (FINGER): study design and progress. Alzheimers Dement. 2013;9(6):657-65 Available from: http://www.ncbi.nlm.nih.gov/ pubmed/23332672.

36. Stephen R, Liu Y, Ngandu T, Antikainen R, Hulkkonen J, Koikkalainen J, et al. Brain volumes and cortical thickness on MRI in the Finnish Geriatric Intervention Study to Prevent Cognitive Impairment and Disability (FINGER). Alzheimers Res Ther. 2019;11(1):1-10.

37. Kivipelto M, Ngandu T, Laatikainen T, Winblad B, Soininen H, Tuomilehto J. Risk score for the prediction of dementia risk in 20 years among middle aged people: a longitudinal, population-based study. Lancet Neurol. 2006; 5(9):735-41 Available from: http://www.ncbi.nlm.nih.gov/pubmed/16914401.

38. Morris JC, Heyman A, Mohs RC, Hughes JP, van Belle G, Fillenbaum G, et al. The Consortium to Establish a Registry for Alzheimer's Disease (CERAD). Part 
I. Clinical and neuropsychological assessment of Alzheimer's disease. Neurology. 1989;39(9):1159-65 Available from: http://www.ncbi.nlm.nih.gov/ pubmed/2771064

39. Harrison J, Minassian SL, Jenkins L, Black RS, Koller M, Grundman M. A neuropsychological test battery for use in Alzheimer disease clinical trials. Arch Neurol. 2007;64(9):1323-9 Available from: http://www.ncbi.nlm.nih.gov/ pubmed/17846273.

40. Jack CR, Wiste HJ, Weigand SD, Knopman DS, Mielke MM, Vemuri P, et al. Different definitions of neurodegeneration produce similar amyloid/ neurodegeneration biomarker group findings. Brain. 2015;138(12):3747-59.

41. Wang Y, Catindig JA, Hilal S, Soon HW, Ting E, Wong TY, et al. Multi-stage segmentation of white matter hyperintensity, cortical and lacunar infarcts. Neuroimage. 2012;60(4):2379-88 Available from: https://doi.org/10.1016/j. neuroimage.2012.02.034.

42. Dzeletovic S, Breuer O, Lund E, Diczfalusy U. Determination of cholesterol oxidation products in human plasma by isotope dilution-mass spectrometry. Anal Biochem. 1995;225(1):73-80 Available from: http://www. ncbi.nlm.nih.gov/pubmed/7778789.

43. Björkhem I, Blomstrand R, Svensson L. Serum cholesterol determination by mass fragmentography. Clin Chim Acta. 1974;54(2):185-93.

44. Picard C, Julien C, Frappier J, Miron J, Théroux L, Dea D, et al. Alterations in cholesterol metabolism-related genes in sporadic Alzheimer's disease. Neurobiol Aging. 2018;66:180.e1-9.

45. Burkard I, von Eckardstein A, Waeber G, Vollenweider P, Rentsch KM. Lipoprotein distribution and biological variation of 24S- and 27hydroxycholesterol in healthy volunteers. Atherosclerosis. 2007:194(1):71-8.

46. Parrado-Fernandez C, Blennow K, Hansson M, Leoni V, Cedazo-Minguez A, Bjorkhem I. Evidence for sex difference in the CSF/plasma albumin ratio in 20 000 patients and 335 healthy volunteers. J Cell Mol Med. 2018;22(10): $5151-4$.

47. Parrado-Fernandez C, Leoni V, Saeed A, Rodriguez-Rodriguez P, SandebringMatton A, Córdoba-Beldad C. et al. Sex difference in flux of 27hydroxycholesterol into the brain. Br J Pharmacol. 2020. https://doi.org/1 0.1111/bph.15353. Epub ahead of print. PMID: 33345295.

48. Heverin M, Maioli S, Pham T, Mateos L, Camporesi E, Ali Z, et al. 27Hydroxycholesterol mediates negative effects of dietary cholesterol on cognition in mice. Behav Brain Res. 2015;278:356-9 Available from: http:// www.ncbinlm.nih.gov/pubmed/25453744.

49. Mast N, Lin JB, Pikuleva IA. Marketed drugs can inhibit cytochrome P450 27A1, a potential new target for breast cancer adjuvant therapy. Mol Pharmacol. 2015;88(3):428-36.

50. Brown J 3rd, Theisler C, Silberman S, Magnuson D, Gottardi-Littell N, Lee JM, et al. Differential expression of cholesterol hydroxylases in Alzheimer's disease. J Biol Chem. 2004;279(33):34674-81.

51. Zhang $X, X i Y, Y u H, A n Y$, Wang $Y$, Tao $L$, et al. 27-Hydroxycholesterol promotes $A B$ accumulation via altering $A B$ metabolism in mild cognitive impairment patients and APP/PS1 mice. Brain Pathol. 2019;29(4):558-73.

52. Popp J, Lewczuk P, Kölsch H, Meichsner S, Maier W, Kornhuber J, et al. Cholesterol metabolism is associated with soluble amyloid precursor protein production in Alzheimer's disease. J Neurochem. 2012;123(2):310-6.

\section{Publisher's Note}

Springer Nature remains neutral with regard to jurisdictional claims in published maps and institutional affiliations.

\section{Ready to submit your research? Choose BMC and benefit from:}

- fast, convenient online submission

- thorough peer review by experienced researchers in your field

- rapid publication on acceptance

- support for research data, including large and complex data types

- gold Open Access which fosters wider collaboration and increased citations

- maximum visibility for your research: over $100 \mathrm{M}$ website views per year

At $\mathrm{BMC}$, research is always in progress.

Learn more biomedcentral.com/submissions 